\title{
DIFERENCIAS EN ALGUNOS PARÁMETROS DEMOGRÁFICOS DE ORYZOMYS CHAPMANI (RODENTIA: MURIDAE) ASOCIADAS A TRES ESTADOS SUCESIONALES DE BOSQUE MESÓFILO DE MONTAÑA EN OAXACA, MÉXICO
}

\author{
Antonio Santos-Moreno', Miguel Ángel Briones-Salas ${ }^{1}$ y Ricardo López-Wilchis ${ }^{2}$ \\ ${ }^{1}$ Centro Interdisciplinario de Investigación para el Desarrollo Integral Regional, Unidad \\ Oaxaca (CIIDIR-OAXACA), Instituto Politécnico Nacional. Calle Hornos 1003, Santa Cruz \\ Xoxocotlán, C. P. 71230 Oaxaca, Oaxaca. MÉXICO. asantosm90@hotmail.com \\ ${ }^{2}$ Departamento de Biología, Universidad Autónoma Metropolitana Unidad Iztapalapa. \\ Apartado Postal 55-535, México 09340, D. F., MÉXICO
}

RESUMEN

Se estudiaron las diferencias en probabilidades de supervivencia y captura, proporción de sexos y
tamaño poblacional en el roedor endémico de México Oryzomys chapmani asociadas a una cronosecuencia
constituida por tres estados sucesionales de bosque mesófilo de montaña. El estudio se realizó en la
región Sierra Norte de Oaxaca y los tiempos de regeneración de los fragmentos de bosque estudiados
fueron 15 (Joven), 40 (Intermedio) y 100 (maduro) años. Se capturaron, marcaron y liberaron 277
ejemplares adultos durante 14 meses. Las probabilidades de supervivencia fueron similares en los
bosques Joven e Intermedio, sin fluctuaciones a través del tiempo, mientras que en el Maduro se observó
un valor más alto en la temporada seca. Las probabilidades de captura no mostraron variación a través del
tiempo en los estados Joven e Intermedio, pero en el Maduro difirieron entre temporadas, con valores más
altos en la de lluvias. Los estados Intermedio y Maduro muestran una proporción $1: 1$ entre sexos, mientras
que el Joven se observó un predominio significativo de machos. El tamaño poblacional vario de 42
individuos por hectárea en la temporada de lluvias a 309 en la seca, ambos en el Bosque Maduro. Palabras Clave: Oryzomys chapmani, supervivencia, proporción de sexos, captura-recaptura, bosque mesófilo, Oaxaca.

\begin{abstract}
We studied the differences among apparent survival and capture probabilities, sex ratio, and population size of the Mexican endemic rodent Oryzomys chapmani associated to a chronosequence integrated by three succesional stages of cloud forest. The study was made at the Sierra Norte de Oaxaca region, and the regeneration times of the forests studied were 15 (Young), 40 (Medium), and 100 (Mature) years. 227 adult specimens were capture, marked, and released by 14 months. The apparent survival probabilities were similar in the Young and Medium forests, without fluctuations across time, whereas in the Mature Forest the dry season shows higher value. The capture probabilities did not show variation across time in the Young and Medium forests, but in the Mature Forest showed difference, with higher value in the rainy season. The Young Forest shows a significant excess of males, whereas the Medium and Mature Forest do not differ significantly from the 1:1 sex ratio. The population size was from 42 individuals by hectares in the rainy season to 302 in the dry season, both in the Mature Forest.
\end{abstract}

Key Words: Oryzomys chapmani, survival, sex ratio, capture-recapture, cloud forest, Oaxaca. 
Santos-Moreno et al.: Parámetros demográficos de un roedor en bosque mesófilo

\section{INTRODUCCIÓN}

El género Oryzomys (Muridae: Sigmodontinae) está representado por 36 especies (Myers et al. 2005), nueve de ellas ocurren en México (Ceballos et al. 2002, RamírezPulido et al. 2005). Aunque los miembros de este género son comunes en varios ambientes de Norte América, los estudios sobre aspectos ecológicos de estas especies son escasos, con excepción de O. palustris, que ha sido estudiada con cierta profundidad, particularmente en ambientes insulares de los Estados Unidos (Forys \& Dueser 1993, Hamilton 1946, Kruchek 2004, Negus et al. 1961, Wolfe 1982). En contraste, las especies que se distribuyen en ambientes tropicales han sido muy poco estudiadas. Una de ellas, Oryzomys chapmani Thomas, 1898, es endémica de México, habita bosques mesófilos de la Sierra Madre Oriental (desde Tamaulipas hasta Veracruz), el Sistema Montañoso (norte de Oaxaca) y Sierra Madre del Sur (sur de Oaxaca y Guerrero) (Musser \& Carleton 2005).

Los bosques mesófilos en México se ubican en climas húmedos, en lugares más húmedos que los habitados por Quercus y más frescos que los habitados por Abies, pero más frescos que aquellos de los boques tropicales (Rzedowski 1991). Debido a las condiciones climáticas particulares que requieren, tienen una distribución limitada y fragmentada. Los bosques nublados de México ocupan aproximadamente el $0.89 \%$ de la superficie territorial del país (SEMARNAP, INEGI \& UNAM 2000). Por unidad de superficie es uno de los más diversos, albergando un número considerable de especies endémicas o amenazadas (Rzedowski 1991). Desafortunadamente también es uno de los ecosistemas mexicanos con mayor grado de amenaza, debido principalmente a las altas tasas de conversión con fines agrícolas. Por ejemplo, de 1971 a 1991 su superficie se redujo a menos del $10 \%$ a una tasa promedio de 78,687 hectáreas por año (Ortega \& Castillo 1996).

Los cambios que sufren las comunidades vegetales en los bosques tropicales de México a través del proceso de sucesión han sido documentados (e.g. Gómez-Pompa \& Vásquez-Yañez 1976), así como las consecuencias de la pérdida de hábitat y de la fragmentación en distintos ambientes en una amplia variedad de taxa (Fahring 2003), incluidas las comunidades de pequeños mamíferos (e.g. Vázquez et al. 1999-2000, García-Estrada et al. 2002). Sin embargo, las diferencias en parámetros demográficos de poblaciones animales que habitan distintos estados sucesionales de vegetación son poco conocidas. Algunos estudios muestran, por ejemplo, como la abundancia de algunas especies de pequeños mamíferos es afectada por la densidad y estratificación del follaje, de acuerdo al grado de madurez de las comunidades vegetales (Malcolm 1995, Pardini 2004, Pardini et al. 2005).

Algunos aspectos de primera importancia en el conocimiento de la demografía de las poblaciones de mamíferos terrestres pequeños son la supervivencia, el tamaño de la población, la proporción de sexos y la estructura de edades. Estos pueden ser estimados a partir de la captura, marcaje, liberación y su recaptura, método conocido simplemente como captura-recaptura. Los primeros estudios de captura-recaptura fueron realizados a principios de la década de 1930 para estimar el tamaño poblacional (e.g., Jackson 1933, 
Lincoln 1930), siendo a partir de la década de 1950 cuando este tipo de estudios se popularizó. La información obtenida con el proceso de captura-marcaje-liberación-recaptura puede ser resumida en una historia de capturas, las cuales son susceptibles de análisis por una amplia variedad de modelos y métodos, que dependiendo de los supuestos en que se basan pueden dividirse en tres grupos (Begon 1979): 1) métodos de enumeración (conteos progresivos de las capturas y recapturas); 2) modelos para poblaciones demográficamente cerradas, en las que se asume que durante las sesiones de trampeo no existe variación en la población por reclutamiento (nacimientos o inmigración) o dilución (mortalidad o emigración (Otis et al. 1978, White et al. 1982); y 3) modelos para poblaciones demográficamente abiertas en las que se asumen y modelan las magnitudes del reclutamiento y la dilución; modelos complejos pero más realistas, por ejemplo, el método probabilistico de Jolly y Sever (Nichols \& Pollock 1983, Schwarz \& Arnason 1996). En México los estudios de poblaciones de mamíferos típicamente emplean métodos de enumeración (e.g. Chávez Tapia \& Gallardo Villegas 1993, Sánchez-Cordero 1993, GarcíaEstrada et al. 2002), en muy pocos se han empleado métodos probabilísticos (e.g. Zalapa et al. 2005). Una ventaja de estos métodos sobre otros como los de enumeración (e.g., el del Mínimo Número de Individuos Vivos propuesto por Krebs en 1966) es que tienen asociadas pruebas para evaluar los efectos de tres fuentes de variación, o bien incorporarlos a modelos específicos: variaciones a través del tiempo, efecto del método de captura y de marca (síndromes de trampofilia y trampofobia) y heterogeneidad debida a que los individuos presentan probabilidades de captura dependientes de su edad, sexo, nivel social o alguna otra característica inherente (Menkens \& Anderson 1988). Los métodos de enumeración no permiten esta evaluación y cuando alguno de estos factores es significativo, no pueden ser incorporados en las estimaciones.

Debido a que se conoce poco como varían los aspectos demográficos de las poblaciones de roedores asociados a distintos estados sucesionales en bosques mesófilos de montaña, el objetivo de este trabajo es conocer las diferencias en probabilidades de supervivencia, de captura, proporción de sexos y tamaño poblacional en Oryzomys chapmani en un bosque mesófilo de montaña con diferente grado de regeneración.

\section{MATERIAL Y MÉTODOS}

Área de Estudio.- La zona de estudio es conocida como El Rincón, en el municipio San Juan Juquila Vijanos (Fig. 1), en la región Sierras Norte de Oaxaca-Mixe. Esta región tiene una alta diversidad de hábitats interconectados y fisiografía compleja, baja fragmentación y la presencia de los bosques mesófilos más extensa y mejor conservados de México (Arriaga-Cabrera et al. 2000). Córdova \& Del Castillo (2001) proporcionan una descripción detallada del área de estudio. La cronosecuencia estudiada está constituida por tres estados sucesionales: 15 años de regeneración (Bosque Joven), 40 años (Bosque Intermedio) y 100 años (Bosque Maduro). Los tiempos de regeneración se estimaron por medio de especies indicadoras (Pinus chiapensis) (Blanco-Macias 2001).

Aunque no se registraron datos climáticos sincrónicos con el trabajo de campo, se compiló una base de datos de las estaciones climáticas del Servicio Meteorológico 
Santos-Moreno et al.: Parámetros demográficos de un roedor en bosque mesófilo

Nacional, a partir de las precipitaciones mensuales de un periodo de 10 años de la estación climatológica Talea de Castro que es la más próxima a la zona de estudio $\left(96.22^{\circ} \mathrm{N}, 17.35^{\circ} \mathrm{W}\right)$ de 1978 a 1984 y $1992-1993$. Estos datos indican que la temporada de lluvias comprende los meses de junio a diciembre (precipitación mensual promedio $189.72 \mathrm{~mm}$, desviación estándar 90.65 ; temperatura media promedio $16.3^{\circ} \mathrm{C}$, desviación estándar 2.33) y alcanza su mayor intensidad de junio a septiembre. La temporada seca va de enero a mayo, con una precipitación mensual promedio de $45.61 \mathrm{~mm}$ (desviación estándar 31.14) y temperatura media promedio de $16.8^{\circ} \mathrm{C}$ (desviación estándar 3.83).

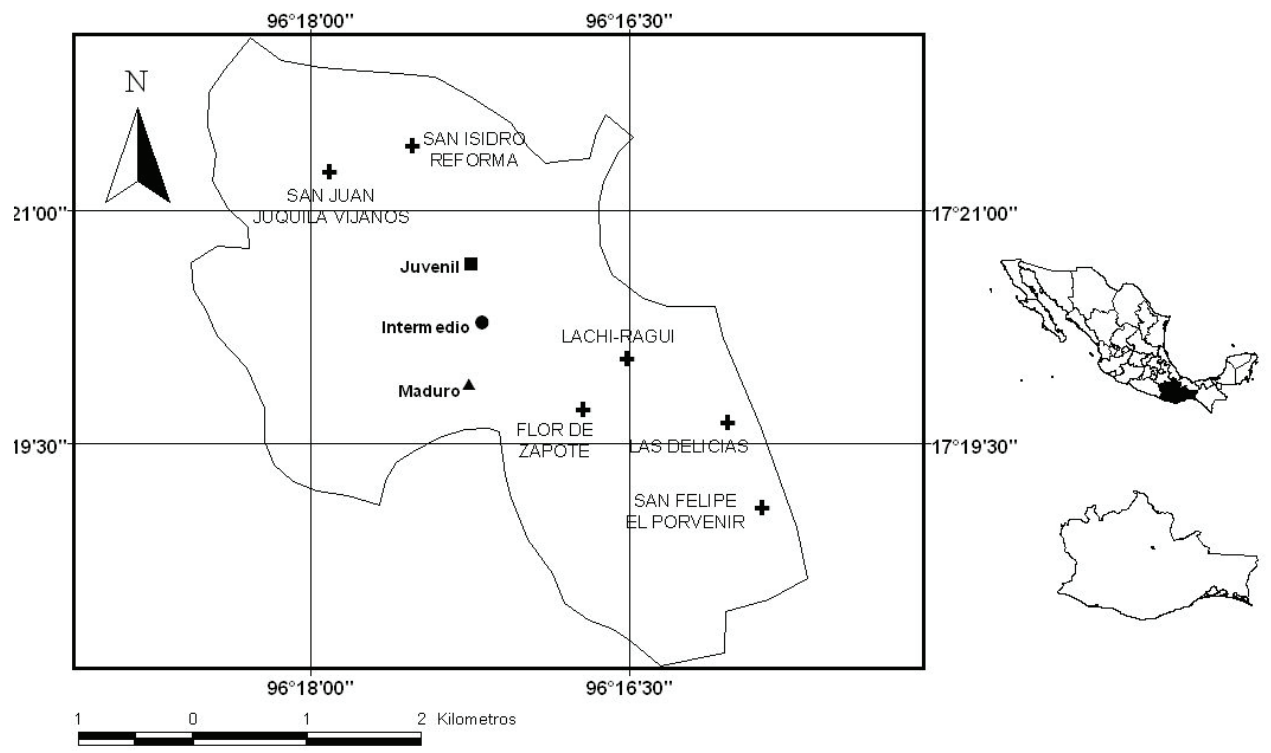

Figura 1

Ubicación geográfica de tres estados sucesionales de bosque mesófilo de montaña en la región de El Rincón, Sierra Norte de Oaxaca, México.

Datos de Campo.- Se realizaron muestreos mensuales en cada estado sucesional, cada uno de cuatro noches. Los bosques Intermedio y Maduro se visitaron 14 meses, desde diciembre de 1999 hasta mayo del 2001. Para el Bosque Joven se contó con datos de 12 meses (de diciembre de 1999 a septiembre del 2000 y de enero a marzo del 2001). De cada estado sucesional se muestrearon dos áreas contiguas de media hectárea cada una, separadas aproximadamente por $300 \mathrm{~m}$. La distancia entre el Bosque Joven y el Intermedio fue de 750 m y entre el Bosque Intermedio y el Maduro de $1.5 \mathrm{~km}$ (Fig. 1). Aunque las distancias son relativamente pequeñas, no se observó el transito de individuos marcados entre estados sucesionales o entre áreas del mismo estado sucesional. Cada área se dividió en una cuadriculas de $10 \mathrm{~m}$, colocando en el vértice de cada cuadro una trampa Sherman $(7.5 \times 7.5 \times 25 \mathrm{~cm})$, dando un total de 66 trampas por área y una hectárea por estado sucesional. Se utilizaron esencia de vainilla y hojuelas de avena 
como cebo. Los ejemplares capturados fueron marcados con el método de ectomización de falanges (Rudran 1996) y liberados inmediatamente en el mismo sitio de la captura.

De cada ejemplar capturado se registró la siguiente información: sexo, condición reproductiva (se consideró como individuos activos en el caso de los machos, a aquellos individuos con testículos escrotados, y en el de las hembras, cuando estaban preñadas o mostraban pezones con leche o con el área periférica carente de pelo. Cuando no se observó ninguna de estas características se registró al individuo como inactivo), edad (jóvenes aquellos individuos de talla pequeña, de pelaje poco denso y de color oscuro, sin evidencias de actividad reproductiva y o adultos) y las medidas corporales convencionales para mamíferos: longitudes máxima corporal, de la cola vertebral, de la pata derecha, de la oreja y el peso. Cuando se trató de ejemplares recapturados se registraron la fecha, el número asignado al ejemplar y la condición reproductiva.

Análisis de Datos.- El tamaño relativamente pequeño de la muestra para cada estado sucesional (73 ejemplares en el Bosque Joven, 87 en el Intermedio y 117 en el Maduro) impidió un análisis por sexos o edad. Por lo tanto, los machos y las hembras adultos se consideraron como un grupo, excluyendo del análisis a los individuos jóvenes.

Las probabilidades de captura $(p)$ y supervivencia $(\Phi)$ para cada estado sucesional se estimaron con el modelo probabilístico de Cormack-Jolly-Sever (Cormack 1964, Jolly 1965, Seber 1965, 1982, 1986). Este modelo permite la posibilidad de cada uno de los parámetros sea constante o bien varíe a través del tiempo (denotado con subíndice $t$, e.g., el modelo en que la probabilidad de supervivencia es constante y la de captura variable se representa como $\Phi p_{t}$ ). El tiempo puede ser expresado de dos formas: como periodos de colecta o agrupando estos en temporadas. La combinación de estas dos condiciones, variación o no de cada uno de los parámetros, y en el segundo caso, el tiempo expresado como periodos de colecta o como temporadas (de lluvias y seca), produjo siete modelos candidatos para explicar la variación de historias de captura en cada estado sucesional (Cuadro 1). La construcción de los modelos y la estimación de los valores de los parámetros se realizó con el programa MARK 4.0 (Cooch \& White 2002). La selección del mejor modelo candidato para cada estado sucesional se realizó con el quasi-Criterio de Información de Akaike (QAIC) (Burnham \& Anderson 2002). Debido a que las historias de captura pueden ser afectadas por factores adicionales a la supervivencia y probabilidad de captura (variación extrabinomial), los valores de los parámetros de todos los modelos fueron ajustados dividiendo su devianza (grado de discrepancia entre el modelo y los datos) entre el promedio de una simulación con 1000 repeticiones.

El modelo probabilístico de Cormack-Jolly-Sever asume que la presencia de individuos transitorios (de paso por la zona, probablemente en proceso de dispersión) no afecta significativamente las estimaciones de supervivencia de los individuos residentes y que el método de captura no tiene un efecto significativo en las probabilidades de recaptura posteriores. Ambos supuestos fueron evaluados empleando el programa U-Care (Choquet et al. 2003). Este programa, a diferencia de otros de uso más común como RELEASE (Burham et al. 1987), tiene implementados algoritmos diseñados para situaciones en que se cuenta con cantidades moderadas de datos y la aplicación de las pruebas es más sencilla. 
Santos-Moreno et al.: Parámetros demográficos de un roedor en bosque mesófilo

La estimación del tamaño poblacional se obtuvo siguiendo el procedimiento propuesto por Lindenmayer et al. (1998) como: $N=n / p$ donde $N$ es el tamaño poblacional estimado, $n$ es el número de animales capturados y $p$ es la probabilidad de captura, estimada a partir del modelo Cormack-Jolly-Seaver. La proporción de sexos para cada estado sucesional se expresó como el número de machos por cada hembra. La significancia de la diferencia entre ésta proporción y una relación 1 a 1 se analizó con la prueba para una proporción basada en coeficientes binomiales (Zar 1999).

\section{Cuadro 1}

Descripción de siete modelos candidatos en tres estados sucesionales de bosque mesófilo de montaña, donde $\Phi=$ probabilidad de supervivencia, $p=$ probabilidad de captura, QAICc=quasi-criterio de información de Akaike corregido, = quasi-verosimilitud (factor de corrección para sobredispersión debida a variación extrabiomial), $\delta Q A I C c=$ diferencias entre QAICc del modelo respectivo y el más parsimonioso, Peso-QAICc=contribución relativa a QAICc del modelo particular al QAICc del conjunto total de modelos.

\begin{tabular}{|c|c|c|c|c|c|c|c|}
\hline $\begin{array}{c}\text { Estado } \\
\text { Sucesional }\end{array}$ & Modelo & QAICc & $\hat{c}$ & ¿QAICc & $\begin{array}{l}\text { Peso- } \\
\text { QAICc }\end{array}$ & $\begin{array}{c}\text { No. de } \\
\text { parámetros }\end{array}$ & QDevianza \\
\hline \multirow[t]{7}{*}{ Joven } & $\Phi p$ & 87.990 & 1.26 & 0 & 0.424 & 2 & 32.426 \\
\hline & $\Phi p_{t(\text { Periodos })}$ & 89.421 & & 1.430 & 0.207 & 11 & 12.480 \\
\hline & $\Phi_{t(\text { Temporadas })} p$ & 90.023 & & 2.032 & 0.1536 & 3 & 32.312 \\
\hline & $\Phi p_{t(\text { Temporadas })}$ & 90.080 & & 2.089 & 0.149 & 3 & 32.370 \\
\hline & $\Phi_{t(\text { Temporadas })} p_{t(\text { Temporadas })}$ & 92.221 & & 4.230 & 0.051 & 4 & 32.312 \\
\hline & $\Phi_{t(\text { Periodos })} p$ & 94.857 & & 6.867 & 0.013 & 11 & 17.916 \\
\hline & $\Phi_{t(\text { Periodos })} p_{t(\text { Periodos })}$ & 102.610 & & 14.620 & 0.0002 & 17 & 8.320 \\
\hline \multirow[t]{7}{*}{ Intermedio } & $\Phi p_{t(\text { Temporadas })}$ & 68.938 & 1.2769 & 0 & 0.356 & 3 & 29.604 \\
\hline & $\Phi p$ & 69.023 & & 0.085 & 0.341 & 2 & 31.822 \\
\hline & $\Phi_{t(\text { Temporadas })} p$ & 70.325 & & 1.386 & 0.178 & 3 & 30.990 \\
\hline & $\Phi_{t(\text { Temporadas })} p_{t(\text { Temporadas })}$ & 71.066 & & 2.128 & 0.123 & 4 & 29.551 \\
\hline & $\Phi p_{t(\text { Temporadas })}$ & 85.157 & & 16.219 & 0.0001 & 14 & 18.837 \\
\hline & $\Phi_{t(\text { Temporadas })} p$ & 87.649 & & 18.710 & 0.00003 & 14 & 21.328 \\
\hline & $\Phi_{t(\text { Temporadas })} p_{t(\text { Temporadas })}$ & 97.334 & & 28.396 & 0 & 20 & 12.912 \\
\hline \multirow[t]{7}{*}{ Maduro } & $\Phi_{t(\text { Temporadas })} p_{t(\text { Temporadas })}$ & 194.951 & 0.9555 & 0 & 0.859 & 4 & 42.319 \\
\hline & $\Phi p_{t(\text { Temporadas })}$ & 199.885 & & 4.934 & 0.072 & 3 & 49.370 \\
\hline & $\Phi p$ & 201.203 & & 6.252 & 0.037 & 2 & 52.775 \\
\hline & $\Phi_{(\text {Temporadas }) t} p$ & 202.879 & & 7.928 & 0.016 & 3 & 52.364 \\
\hline & $\Phi p_{t(\text { Periodos })}$ & 204.122 & & 9.171 & 0.008 & 14 & 28.498 \\
\hline & $\Phi_{t(\text { Periodos })} p$ & 205.408 & & 10.457 & 0.004 & 14 & 29.785 \\
\hline & $\Phi_{t(\text { Periodos })} p_{t(\text { Periodos })}$ & 216.159 & & 21.208 & 0.00002 & 22 & 19.383 \\
\hline
\end{tabular}

\section{RESULTADOS}

Se capturaron, marcaron y liberaron 277 ratones adultos. El promedio de capturas por ejemplar fue bajo en los tres estados sucesionales y no se observaron diferencias significativas entre ellos: a continuación se muestra el valor promedio y entre paréntesis los límites del intervalo del 95 \% de confianza para la media: Bosque Joven 1.29 (1.101.47), Bosque Intermedio 1.17 (1.01-1.33), Bosque Maduro 1.33 (1.19-1.47). 
Selección de Modelos.- Para el Bosque Joven después de 1000 simulaciones solo 298 mostraron una devianza mayor que la observada en el modelo con el valor más bajo de QAIC. Después de corregir los valores por sobredispersión el mejor modelo fue el reducido $(\Phi p)$ esto es, aquel en el que tanto la supervivencia como las probabilidades de captura son constantes (Cuadro 1). Este modelo es apoyado por los datos en más de $450 \%$ que el segundo mejor modelo candidato.

Para el Bosque Intermedio 198 simulaciones mostraron valores de devianza mayores a la observada para el mejor modelo candidato. Después del ajuste para eliminar el efecto de la sobredispersión el mejor modelo fue $\Phi p_{t}$, con el tiempo agrupado en estaciones, aunque las historias de captura apoyan este modelo solo ligeramente en grado mayor (104 \%) que el reducido. Debido a que ambos modelos están anidados (i.e., la transformación de un modelo en el otro requiere de la adición o sustracción de uno o más factores, pero no ambas, en este caso la dependencia del tiempo), se aplicó una prueba de relaciones de verosimilitud, no encontrando diferencias estadísticamente significativas entre ambos $\left(X^{2}=2.219, \mathrm{gl}=1, \mathrm{P}=0.14\right)$, por lo que seleccionamos al modelo reducido como el más parsimonioso (Cuadro 1 ).

Para el Bosque Maduro 556 de las 1000 simulaciones mostraron devianzas mayores que el mejor modelo candidato. Los valores ajustados por sobredispersión indican que el modelo completo $\left(\Phi_{t} p_{t}\right)$, con el tiempo expresado como temporadas, es el que mejor explica el patrón de las historias de captura en este estado sucesional. Este modelo fue apoyado fuertemente por los datos, once veces más que el segundo mejor modelo. La prueba de relación de verosimilitudes muestra diferencias significativas $\left(X^{2}=7.05, \mathrm{gl}=\right.$ $1, P=0.008)$ entre el modelo completo y el segundo mejor modelo $\left(\Phi p_{t}\right)$, por lo que consideramos el primero como el más adecuado.

Tamaño Poblacional.- En el Bosque Joven se marcaron 73 ejemplares, de los cuales solo el $18 \%$ se capturó en dos ocasiones, $1 \%$ en tres y $1.36 \%$ en siete periodos. No se observó efecto estadísticamente significativo de individuos transitorios $\left(X^{2}=-0.292, P\right.$ $=0.77)$ ni efecto del trampeo $\left(X^{2}=0, P=1\right)$. El tamaño poblacional estimado fue de 169 individuos/ha (Cuadro 2).

En el Bosque Intermedio se marcaron 87 ejemplares; el $90.5 \%$ de ellos se capturaron una ocasión, $5 \%$ en dos y $2.35 \%$ en tres. La máxima permanencia la mostró un ejemplar capturado en siete periodos consecutivos. No se detectó efecto significativo de individuos transitorios $\left(X^{2}=1.28, P=0.19\right)$ ni del trampeo $\left(X^{2}=-0.96, P=0.33\right)$. Se estimo el tamaño poblacional como 230 individuos por hectárea.

Para el Bosque Maduro la muestra estuvo constituida por 117 ejemplares, $77.77 \%$ de los cuales se capturó en una ocasión, $19 \%$ en dos, y el tiempo máximo de permanencia se observó en dos ejemplares, los cuales fueron capturados en cinco periodos. No hubo diferencias significativas debidas a individuos transitorios $\left(X^{2}=0.07\right.$, $P=0.47) \circ$ al trampeo $\left(X^{2}=-0.51, P=0.60\right)$. Para la temporada seca se estimó un tamaño poblacional de 309 individuos por hectárea, mientras que en la de lluvias fue de 42 (Cuadro 2). 
Santos-Moreno et al.: Parámetros demográficos de un roedor en bosque mesófilo

Probabilidad de Captura.- Para el Bosque Joven de acuerdo al mejor modelo la probabilidad de captura promedio de ejemplares es baja, 0.46 , para el Bosque Intermedio fue 2.5 veces menor, los intervalos de confianza del $95 \%$ construidos a partir de las desviaciones estándar, muestran que no existen diferencias significativas entre los bosques Joven e Intermedio (Cuadro 1). Para el Bosque Maduro fluctuaron de 0.275 en la temporada seca a 0.99 en la de lluvias (Cuadro 2).

\section{Cuadro 2}

Probabilidades de captura $(p)$, supervivencia $(\Phi)$, número de ejemplares capturados $(n)$ y tamaño poblacional estimado $(N)$ de Oryzomyz chapmani de acuerdo al mejor modelo para tres estados sucesionales de boque mesófilo de montaña. Para cada parámetro se muestra el valor y entre paréntesis los límites del intervalo al $95 \%$ de confianza.

\begin{tabular}{|c|c|c|c|}
\hline $\begin{array}{c}\text { Estado } \\
\text { Sucesional }\end{array}$ & Modelo Final & Parámetro & $\begin{array}{c}\text { Valor } \\
\text { (Intervalos del } 95 \% \text { de } \\
\text { confianza) }\end{array}$ \\
\hline \multirow[t]{4}{*}{ Joven } & $\Phi p$ & $p$ & $0.426(0.164-0.737)$ \\
\hline & & $\Phi$ & $0.369(0.205-0.571)$ \\
\hline & & $n$ & 72 \\
\hline & & $N$ & 169 \\
\hline \multirow[t]{4}{*}{ Intermedio } & $\Phi p$ & $p$ & $0.169(0.048-0.452)$ \\
\hline & & $\Phi$ & $0.411(0.195-0.667)$ \\
\hline & & $n$ & 85 \\
\hline & & $N$ & 230 \\
\hline \multirow[t]{8}{*}{ Maduro } & $\Phi_{t(\text { Temporadas })} p_{t(\text { Temporadas })}$ & $p_{\text {Temporada seca }}$ & $0.275(0.133-0.484)$ \\
\hline & & $p_{\text {Temporada de lluvias }}$ & $0.989(0.989-1)$ \\
\hline & & $\Phi_{\text {Temporada seca }}$ & $0.509(0.319-0.696)$ \\
\hline & & $\Phi_{\text {Temporada de lluvias }}$ & $0.212(0.134-0.318)$ \\
\hline & & $n_{\text {Temporada seca }}$ & 85 \\
\hline & & $n_{\text {Temporada de lluvias }}$ & 42 \\
\hline & & $N_{\text {Temporada seca }}$ & 309 \\
\hline & & $N_{\text {Temportada de lluvias }}$ & 42 \\
\hline
\end{tabular}

Probabilidad de Supervivencia - En el Bosque Joven se obtuvo un valor de 0.369 , en el Bosque Intermedio se observó un valor un poco más alto que en el Bosque Joven (0.411), al igual que para las probabilidades de captura, los intervalos del $95 \%$ de confianza indican que las diferencias no son significativas en este parámetro entre los bosques Joven e Intermedio. En el Bosque Maduro para la temporada seca el valor fue de 0.509 y 0.2124 para la temporada lluviosa.

Proporción de Sexos.- En el Bosque Joven se registraron 27 hembras y 46 machos, que determinan una proporción de 1:1.7, que difirió significativamente de 1:1 $\left(X^{2}=4.94\right.$, 
$\mathrm{gl}=1, \mathrm{P}=0.02)$. En el Bosque Intermedio se registraron 35 hembras y 52 machos, que determinan una proporción de sexos de 1:1.48, que no es estadísticamente diferente de $1: 1\left(X^{2}=3.32, \mathrm{gl}=1, P=0.06\right)$. Para el Bosque Maduro la muestra estuvo constituida por 57 hembras y 60 machos, que determinan una proporción de 1:1.05, que no difiere significativamente de $1: 1\left(\mathrm{X}^{2}=0.7815, \mathrm{gl}=1, \mathrm{P}=0.78\right)$. Para la temporada seca se obtuvo un tamaño poblacional de 309 individuos por hectárea, mientras que en la de lluvias fue de 42 (Cuadro 2).

\section{DISCUSIÓN}

Supervivencia.- La actividad reproductiva de O. chapmani en la zona de estudio ocurre principalmente entre febrero y mayo, con algunos eventos esporádicos en agosto. Si se toma como referencia el mes de abril, y que en promedio los ejemplares fueron recapturados 1.26 ocasiones (meses) a partir de diciembre, que es el mes en que se inició el muestreo, se tiene que la vida promedio de un adulto es de 11 a 12 meses, sin diferencias significativas entre estados sucesionales y con un valor máximo de 15 meses. El valor promedio de 11 a 12 meses es similar a lo observado en Peromyscus maniculatus, para el que se indica que del 94 al $99 \%$ de la población no sobrevive más de un año (Terman 1968), aunque para Neotomodon alstoni en una zona templada del centro de México Chávez Tapia y Gallardo Villegas (1993) encontraron que la supervivencia del 50 $\%$ de los adultos estuvo entre 75 a 105 días. Para Heteromys desmarestianus solo el 20 $\%$ del total de individuos capturados permanecieron por tres meses o más dentro del área de estudio (Sánchez-Cordero 1993), aunque esta estimación incluyó a ejemplares jóvenes que eventualmente se dispersan a otras zonas.

Las diferencias observadas en las probabilidades de supervivencia pueden ser vistas como flexibilidad demográfica, similar a la que se ha observado en otras especies de mamíferos en ambientes naturalmente fragmentados y puede ser un importante atributo que permite la persistencia en hábitat de calidad pobre (Adler \& Wilson 1987, Dickman \& Doncaster 1989). Por ejemplo, en los estados sucesionales Joven e Intermedio el modelo indicó que la supervivencia es constante a través del tiempo, mientras que en el Maduro existen diferencias entre estaciones, con un promedio de 0.36 . Los valores indican una relación inversamente proporcional entre supervivencia y grado de madurez del Bosque. Otros estudios muestran relaciones diversas, lo que puede indicar sensibilidad diferencial de las especies a los efectos de la perturbación. Por ejemplo en Peromyscus mexicanus las variaciones en la densidad poblacional corresponde mejor a cambios climáticos que a perturbaciones debidas a actividades humanas (Martínez-Gallardo 1995), mientras que Apodemus sylvaticus muestra mayor supervivencia en zonas sin disturbios (Dickman \& Doncaster 1989).

Por otra parte, los parámetros demográficos pueden ser afectados por la disponibilidad de recursos. Por ejemplo, Adler y Wilson (1987) desarrollaron un modelo simple que relaciona la demografía con lo adecuado del ambiente. De acuerdo con este modelo la supervivencia y otros parámetros se incrementan a lo largo de un gradiente de ade- 
Santos-Moreno et al.: Parámetros demográficos de un roedor en bosque mesófilo

cuación ambiental. Asumiendo que en el Bosque Maduro se presenten las condiciones más adecuadas para $O$. chapmani, nuestros resultados concuerdan con este modelo, mostrando un incremento de las probabilidades de supervivencia entre el estado sucesional Joven (0.369) y los estados Intermedio (0.411) y Maduro, solo si se promedian los valores de las temporadas seca y de lluvias (0.509 y 0.212 , respectivamente).

Para otras especies de roedores de zonas templadas como por ejemplo Phyllotis darwini se sabe que las fluctuaciones climáticas tienen efecto en distintos aspectos demográficos (Lima et al. 2001), mientras que para aquellos que habitan en zonas más cálidas como Heteromys desmarestianus se ha observado que el tamaño poblacional y la reproducción están limitados por la disponibilidad de alimento (Sánchez-Cordero 1993).

Probabilidades de Captura.- Las probabilidades de captura pueden ser un reflejo de la cantidad de movimiento de los individuos, lo que a su vez puede deberse a la necesidad de buscar alimento o bien una pareja reproductiva. La primera explicación no se ve apoyada por nuestros resultados, ya que el Bosque Maduro es el único estado sucesional en que se observó que las probabilidades de captura difieren entre temporadas, sin embargo, el patrón es contrario a lo esperado si la disponibilidad de alimento fuera el factor determinante de la movilidad y eventual probabilidad de captura: en la temporada seca en que disminuye la cantidad de alimento se esperaría que en ella la supervivencia disminuyera y las probabilidades de captura se incrementaran, pero las estimaciones de los parámetros muestran el patrón contrario.

La alternativa de alta movilidad y por lo tanto, mayor probabilidad de captura asociada a la actividad reproductiva parece ser coherente con los valores observados de mayor probabilidad de captura en la temporada seca que en la de lluvias. En este estudio la mayor cantidad de evidencias de actividad reproductiva, particularmente machos con testículos escrotados, se observó en los meses de febrero y mayo en los estado sucesionales Intermedio y Maduro, aunque en el estado Joven también se observaron a la mitad de la temporada de lluvias en el mes de agosto, aunque con baja frecuencia. Se capturaron ejemplares jóvenes principalmente en febrero y mayo y algunos en junio y agosto. Este patrón de máxima actividad reproductiva y nacimiento de las crías en la temporada más favorable a inicios de la temporada de lluvias se ha observado en otras especies y es una estrategia que maximiza la disponibilidad de alimentos para los individuos jóvenes (Chávez Tapia \& Gallardo Villegas 1993). Para varias especies de Peromyscus se ha registrado el mismo patrón de actividad reproductiva elevada a partir de la primavera y durante el verano, coincidiendo con los meses de mayor número de horas luz (Banks 1967, MacMillen 1964).

Proporción de Sexos.- La desviación de la proporción de sexos de 1:1 con exceso de machos observado únicamente en el estado sucesional Joven parece ser una respuesta común de algunas especies de roedores a las perturbaciones, aunque el predominio de machos o hembras no parece una tendencia clara. Estudios en Peromyscus aztecus y Reithrodontomys fulvescens en bosques mesófilos de montaña en el estado de Jalisco (Vázquez et al. 1999-2000) y Oryzomys palustris en ambientes templados (Joule \& 
Jameson 1972, Park \& Nowsielski-Slepowron 1972) muestran el mismo patrón. Adiferencia de estos estudios, en una selva baja caducifolia no se observaron diferencias en proporción de sexos en seis especies de roedores (no se registró a $O$. chapmani entre ellas) en hábitat perturbados y no perturbados en la sierra de Huautla, en el estado de Morelos (García-Estrada et al. 2002). Para O. longicaudatus en el sur de Chile se encontró que la proporción de sexos está sesgada hacia los machos (Murúa et al. 1986). La proporción de sexos 1:1 ha sido señalada para Peromyscus maniculatus y P. leucopus (Gounddie \& Vessey 1986, Wolff \& Durr 1986).

Tamaño Poblacional.- Como ya se mencionó, no existen muchos estudios con la aplicación de métodos de captura-recaptura para especies del género Oryzomys. En uno de ellos sobre $O$. capito en bosques de Panamá, al contrario de este estudio, se observó la densidad más alta en la temporada de lluvias (de 3.21 a 4.48 individuos por hectárea), mientras que en la seca fue de 0.247 (Fleming 1970). Las estimaciones anteriores parecen muy bajas en comparación con las obtenidas en este estudio, particularmente con las del estado Maduro en temporada seca (309 individuos por hectárea), pero existen dos factores que pueden explicarlas. En primer lugar, las estimaciones de Fleming (1970) son el resultado de la división del número de ejemplares capturados por unidad de superficie, mientras que en este estudio el número de individuos capturados en la temporada seca fue de 85 , lo que incrementa la estimación es la división entre probabilidad de captura, la cual no es incluida en la estimación de Fleming (1970). El uso de los conteos totales presenta los mismos sesgos que las estimaciones de tamaño poblacional por el método MNIV, aunque no tiene el problema de la desigualdad del sesgo en los periodos iniciales y finales.

Por otra parte, debido a que en este estudio el modelo que mejor explicó la variación en las historias de captura en el Bosque Maduro fue aquel en que no existen variaciones significativas entre los meses que integran cada temporada, por lo que el número de individuos capturados corresponde a la suma de las capturas en los seis meses correspondientes a dicha estación. A diferencia de esto, Fleming (1970) menciona únicamente dos meses para cada una de sus estimaciones.

Es probable que al considerar las agrupaciones temporales y la ponderación entre probabilidades de captura muchas de las estimaciones de estudios basados en métodos de enumeración arrojen estimaciones similares a las aquí mencionadas. Por ejemplo, para Oryzomys alfaroi y otras cuatro especies de roedores en una selva tropical en la Reserva de Biología Tropical Chamela en el estado de Jalisco (Sánchez-Cordero et al. 1997) presentan datos de tamaño poblacional y algunos datos sobre recapturas. Desgraciadamente para esta especie no muestran probabilidades de captura, por lo que no es factible una comparación directa con los resultados aquí obtenidos, pero sí se toman los datos de Peromyscus mexicanus del mismo estudio, resulta que el número máximo de ejemplares capturados es 77 , mientras que la probabilidad de captura para la misma sesión fue de 0.22 , lo que da un total de 250 individuos por hectárea en una sola sesión (noche) de trampeo. 
Santos-Moreno et al.: Parámetros demográficos de un roedor en bosque mesófilo

Existen otros factores que aunque no fueron evaluados en este estudio se sabe que afectan aspectos demográficos de los animales. Una de estas son las características físicas de los fragmentos, tales como su tamaño y forma, que indirectamente actúan sobre las poblaciones en diferentes formas. Como ya se mencionó, una de las características de los bosques mesófilos es que se encuentran naturalmente fragmentados, a lo que se adiciona el efecto de las actividades antropogénicas. Las condiciones ambientales pueden variar con el tamaño del fragmento (Sousa 1984), por lo que las subpoblaciones que habitan diferentes regiones del fragmento pueden diferir en estructura de edades, composición genética y característica de las historias de vida (Thompson 1984).

\section{AGRADECIMIENTOS}

El presente trabajo es parte de los resultados de la tesis del primer autor para obtener el grado de Doctor en el Programa de Doctorado en Ciencias Biológicas de la Universidad Autónoma Metropolitana (UAM), México. La Secretaría de de Educación y Posgrado del Instituto Politénico Nacional, el Consejo Nacional de Ciencia y Tecnología y la Darwin Initiative for the Survival of the Species otorgaron apoyo económico. Y. Hernández, A. L. Trujano, R. Rivera, J. Calderón y C. L. García proporcionaron apoyo para el trabajo de campo. A. Alfaro y dos revisores anónimos hicieron valiosos comentarios y sugerencias al manuscrito.

\section{LITERATURA CITADA}

Adler, G. H. \& M. L. Wilson. 1987. Demography of habitat generalist, the white-footed mouse, in a heterogeneous environment. Ecology 68: 1785-1796.

Arriaga Cabrera, L., J. M. Espinoza-Rodriguez, C. Aguilar-Zuñiga, E. Martínez-Romero, L. Gómez-Mendoza \& E. Loa Loza (Coords.). 2000. Regiones Terrestres Prioritarias de México. Comisión Nacional para el Conocimiento y Uso de la Biodiversidad. México. $608 \mathrm{p}$.

Banks, R. C. 1967. The Peromyscus guardia-interparietalis complex. J. Mamm. 48: 210-218.

Begon, B. 1979. Investigating Animal Abundance: Capture-Recapture for Biologists. University Park Press, Baltimore. $97 \mathrm{p}$.

Blanco Macias, A. M. 2001. Análisis sucesional del bosque mesófilo de montaña en El Rincón, Sierra Norte de Oaxaca. Tesis Profesional, Facultad de Estudios Superiores Iztacala, UNAM, México.

Burnham, K. P. \& D. R. Anderson. 2002. Model Selection and Inference -A Practical Information- Theoretic Approach. Springer-Verlag, New York. $488 p$

Burnham, K. P., D. R. Anderson, G. C. White, C. Brownie \& K. H. Pollock. 1987. Design and Analysis Methods for Fish Survival Experiments Based on Release-Recapture. Amer. Fish. Soc. Monogr. 5: 1-436.

Ceballos, G., J. Arroyo-Cabrales \& R. A. Medellin. 2002. The mammals of Mexico: composition, distribution, and conservation status. Occas. Papers Mus. Texas Tech Univ. 218: 1-27.

Chávez Tapia, C. B. y R. Gallardo Villegas. 1993. Demografía y reproducción de Neotomodon alstoni en la Sierra del Ajusco, México. Pp. 317-334, In: R. A. Medellín \& G. Ceballos (Eds.). Avances en el Estudio de los Mamíferos de México. Asociación Mexicana de Mastozología, A. C. Publ. Esp. 1: 1-464. 
Choquet, R., A. M. Reboulet, R. Pradel, O. Gimenez \& J. D. Lebreton. 2003. User's Manual for U-Care, Utilities-CApture-REcapture, V2.02, CEFE/CNRS, Montpellier (ftp://ftp.cefe.cnrs.fr/ biom/U-CARE).

Cooch, E. \& G. White. 2002. Program MARK. Analysis of Data from Marked Individuals. A Gentle Introduction. 2d. ed. Ithaca, New York. 284 p.

Córdova, J. \& R. Del Castillo. 2001. Changes in epiphyte cover in three cronosequences in a tropical montane cloud forest in Mexico. Pp. 79-94, In: G. Gottsberger \& S. Liede (Eds.). Life Forms and Dynamics in Tropical Forests. J. Cramer Gerbrüder Borntraeger Verlagsbuchhandlung. Berlin.

Cormack, R. M. 1964. Estimates of survival from the sighting of marked animals. Biometrika 51: 429-438.

Dickman, C. R. \& C. P. Doncaster. 1989. The ecology of small mammals in urban habitats. II. Demography and dispersal. J. Anim. Ecol. 58: 119-127.

Fahring, L. 2003. Effects of habitat loss and fragmentation on biodiversity. Ann. Rev. Ecol. Syst. 34: 487-515.

Fleming, T. H. 1970. Comparative biology of two temperate-tropical rodent counterparts. Am. Midl. Nat. 83: 462-471.

Forys, E. A. \& R. D. Dueser. 1993. Inter-island movements of rice rats (Oryzomys palustris). Am. Midl. Nat. 130: 408-412.

García-Estrada, C., M. L. Romero-Almaráz \& C. Sánchez-Hernández. 2002. Comparison of rodent communities in sites with different degrees of disturbance in deciduous forest of southeastern Morelos, Mexico. Acta Zool. Mex. (n. s.) 85: 153-168.

Gómez-Pompa, A. \& C. Vásquez-Yañez. 1976. Estudios sobre sucesión secundaria en los trópicos cálido-húmedos: el ciclo de vida de las especies secundarias. Pp. 579-593, In: A. Gómez-Pompa, C. Vásquez-Yañes, R. S. del Amo \& A. C. Butanda (Eds.). Investigaciones Sobre la Regeneración de las Selvas Altas en Veracruz, México. Consejo Nacional para la Enseñanza de la Biología.

Gounddie, T. \& S. H. Vessey. 1986. Survival and dispersal of young white-footed mice born in nest boxes. J. Mamm. 67: 53-59.

Hamilton, W. J., Jr. 1946. Habits of swamp rice rat Oryzomys palustris palustris (Harlan). Am. Midl. Nat. 36: 730-736.

Jackson, C. H. N. 1933. On the true density of tsetse flyes. J. Anim. Ecol. 2: 204-209.

Jolly, G. M. 1965. Explicit estimates from capture-recapture data with both death and immigration-stochastic model. Biometrika 52: 225-247.

Joule, J. \& D. L. Jameson. 1972. Experimental manipulation of population density in three sympatric rodents. Ecology 53: 653-660.

Krebs, C. J. 1966. Demographic changes in fluctuating populations of Microtus californicus. Ecol. Monogr. 36: 239-73.

Kruchek, B. L. 2004. Use of tidal marsh and upland habitats by the marsh rice rat (Oryzomys palustris). J. Mamm. 85: 569-575.

Lima, M., R. Julliard, N. Chr. Stenseth \& F. M. Jaksic. 2001. Demographic dynamics of a neotropical small rodent (Phillotis darwini): feedback structure, predation and climatic factors. J. Anim. Ecol. 70: 761-775.

Lincoln, F. C. 1930. Calculating Waterfowl Abundance on the Basis of Ganding Returns. Circular of the U. S. Department of Agriculture No. 118: 1-4.

Lindenmayer, D. B., R. C. Lacy \& K. L. Viggers. 1998. Modelling survival and capture probabilities of the mountain brushtail possum (Trichosurus caninus) in the forests of southeastern Australia using trap-recapture data. J. Zool. 245: 1-13. 
Santos-Moreno et al.: Parámetros demográficos de un roedor en bosque mesófilo

MacMillen, R. E. 1964. Population ecology, water relations, and social behavior of a southern California semidesert rodent fauna. Univ. California Publ., Zool. 71: 1-66.

Malcolm, J. R. 1995. Forest structure and the abundance and diversity of Neotropical small mammals. Pp. 179-197, In: M. D. Lowman \& N. M Nadkarni (Eds.). Forest Canopies Academic Press. New York.

Martínez-Gallardo, R. 1995. Remoción postdispersión de semillas y frutos por mamíferos en diferentes grados de perturbación antropogénica de la selva alta perennifolia de la región de Los Tuxtlas, Veracruz. Tesis Doctoral, Facultad de Ciencias, UNAM, México, 116 p.

Menkens, G. E., Jr. \& S. H. Anderson. 1988. Estimation of small mammal population size. Ecology 69: 1952-1959.

Murúa, R., L. A. González \& P. L. Meserve. 1986. Population ecology of Oryzomys longicaudatus philippii (Rodentia: Cricetidae) in southern Chile. J. Anim. Ecol. 55: 281-293.

Musser, G. C. \& M. D. Carleton. 2005. Superfamily Muroidea. Pp. 894-1531, In: D. E. Wilson \& D. A. M. Reeder (Edits.). Mammal Species of the World. Taxonomic and Geographic Reference 3a. ed. The Johns Hopkins University Press. Baltimore. xxxv+1-743 + xvii+745$2142 \mathrm{pp}$.

Myers, P., R. Espinosa, C. S. Parr, T. Jones, G. S. Hammond \& T. A. Dewey. 2005. The Animal Diversity Web (online). Acceso Noviembre de 2005. URL:http://animaldiversity.org.

Negus, N. C., E. Gould \& R. K. Chipman. 1961. Ecology of the rice rat, Oryzomys palustris (Harlan), on Breton Island, Gulf of Mexico, with a critique of social stress theory. Tulane Stud. Zool. 8: 3-123.

Nichols, J. D. \& K. H. Pollock. 1983. Estimation methodology in contemporary small mammal capture-recapture studies. J. Mamm. 64: 256-261.

Ortega, E. S. \& C. G. Castillo. 1996. El bosque mesófilo de montaña y su importancia forestal. Ciencias 43: 32-39.

Otis, D. L., K. P. Burnham, G. C. White \& D. R. Anderson. 1978. Statistical inference from capture data on closed animal populations. Wildl. Monogr. 62: 1-137.

Pardini, R. 2004. Effects of forest fragmentation on small mammals in an Atlantic Forest landscape. Biodiv. Conserv. 13: 2567-2586,

Pardini, R., S. Marques de Souza, R. Braga-Neto \& J. P. Metzger. 2005. The role of forest structure, fragment size and corridors in maintaining small mammal abundance and diversity in an Atlantic forest landscape. Biol. Conserv. 124: 253-566.

Park, A. W. \& B. J. A. Nowsielski-Slepowron. 1972. Biology of the rice rat (Oryzomys palustris natator) in a laboratory environment. Z. Saugetierk. 37: 42-51.

Ramírez-Pulido, J., J. Arroyo-Cabrales \& A. Castro-Campillo. 2005. Estado actual y relación nomenclatural de los mamíferos terrestres de México. Acta Zool, Mex. (n. s.) 21: 21-82.

Rudran, R. 1996. Methods for marking mammals. Pp. 299-310, In: D. E. Wilson, F. R. Cole, J. D. Nichols, R. Rudran \& M. S. Foster (Eds.). Measuring and Monitoring Biological Diversity: Standard Methods for Mammals. Smithsonian Institution Press, Washington, D. C. XXVII+409 p.

Rzedowski, J. 1991. Diversidad y orígenes de la flora fanerógamica de México. Acta Bot. Mex. 14: 3-22.

Sánchez-Cordero, V. 1993. Estudio poblacional de la rata espinosa Heteromys desmarestianus en la selva húmeda en Veracruz, México. Pp. 301-316, In: R. A. Medellín \& G. Ceballos (Eds.). Avances en el Estudio de los Mamíferos de México. Asociación Mexicana de Mastozología, A. C. Publ. Esp. 1: 1-464. 
Sánchez.Cordero, V., G. Magaña C. \& M. A. Briones. 1997. Modelos de captura y recaptura en cinco especies de roedores. Pp. 297-324, In: J. Arroyo-Cabrales y O. J. Polaco (Coords.). Homenaje al Profesor Ticul Álvarez Inst. Nal. Antropología e Hist., Colección Científica. $391 \mathrm{p}$.

Schwarz, C. J. \& A. N. Arnason. 1996. A general methodology for the analysis of capturerecapture experiments in open populations. Biometrics 52: 860-873.

Seber, G. A. F. 1965. A note in multiple recapture census. Biometrika 52: 249-259. . 1982. The Estimation of Animal Abundance and Related Parameters. Charles Griffin \& Co., London. .1986. A review of estimating animal abundance. Biometrics 42: 267-292.

Secretaria de Medio Ambiente Recursos Naturales y Pesca (SEMARNAP), Instituto Nacional de Estadística, Geografía e Informática (INEGI) e Instituto de Geografía, Universidad Nacional Autónoma de México (UNAM). 2000. Inventario Forestal Nacional 2000-2001. Escala 1:250000. Instituto de Geografía, UNAM, México.

Sousa, W. P. 1984. The role of disturbance in natural communities. Ann. Rev. Ecol. Syst. 15: 353-391.

Terman, C. R. 1968. Population dynamics. Pp. 412-445. In: J. A. King (Ed.). Biology of Peromyscus (Rodentia: Cricetidae). Spec. Publ., Amer. Soc. Mamm. 2: 1-593

Thompson, J. M. 1984. Within-patch dynamics of life histories, populations and interactions: selection over time in small spaces. Pp. 253-265, In: S. T. A. Pickett \& P. S. White (Edits.). Natural Disturbance: The Patch Dynamics Perspective. Academic Press. New York. 472 p.

Vázquez, L. B., G. N. Cameron \& R. A. Medellín. 1999-2000. Hábitos alimentarios y biología poblacional de dos especies de roedores en el occidente de México. Rev. Mex. Mastozool. 4: 5-21.

White, G. C., D. R. Anderson, K. P. Burnham \& D. L. Otis. 1982. Capture-recapture and removal methods for sampling closed populations. Los Alamos National Laboratory LA-8787NERP. Los Alamos, Nuevo Mexico.

Wolfe, J. L. 1982. Oryzomys palustris. Mammalian Species 176: 1-5.

Wolff, J. O. \& D. S. Durr. 1986. Winter nesting behavior of Peromyscus leucopus and Peromyscus maniculatus. J. Mamm. 67: 409-412.

Zalapa, S. S., M. H. Badii, F. A. Cervantes \& S. Guerrero. 2005. Ecología poblacional de Liomys pictus en tres áreas de bosques tropical subcaducifolio con diferente tiempo de regeneración, en la costa Norte de Jalisco, México. Acta Zool. Mex. (n. s.) 21: 1-14.

Zar, H. H. 1999. Biostatistical Analysis. $4^{a}$. ed. Prentice Hall, Upper Saddle River, New Jersey. $\mathrm{xii}+663+212+11+23 \mathrm{p}$.

Recibido: 15 de enero 2006

Aceptado: 14 de octubre 2006 
